ISSN 0010-5082, Combustion, Explosion, and Shock Waves, 2018, Vol. 54, No. 2, pp. 158-164. (c) Pleiades Publishing, Ltd., 2018.

Original Russian Text (C) A.E. Ligachev, G.V. Potemkin, O.K. Lepakova, M.V. Zhidkov, A.D. Teresov, N.N. Golobokov, Yu.M. Maksimov,

Yu.R. Kolobov, N.N. Koval'.

\title{
Ignition of a Ti-Al-C System by an Electron Beam
}

\author{
A. E. Ligachev ${ }^{a}$, G. V. Potemkin ${ }^{b}$, O. K. Lepakova ${ }^{c}$, \\ UDC 541.126 \\ M. V. Zhidkov ${ }^{d, e}$, A. D. Teresov ${ }^{b, f}$, N. N. Golobokov ${ }^{c}$, \\ Yu. M. Maksimov ${ }^{c}$, Yu. R. Kolobov ${ }^{d, e}$, and N. N. Koval ${ }^{b, f}$
}

Published in Fizika Goreniya i Vzryva, Vol. 54, No. 2, pp. 39-45, March-April, 2018.

Original article submitted May 19, 2017.

\begin{abstract}
This paper describes the optimal modes of initiation of self-propagating hightemperature synthesis with the help of an electron beam on the example of a Ti-Al-C powder mixture. A pulsed electron beam with a particle energy of tens of kiloelectronvolts and a duration of hundreds of microseconds is used. Morphology, structure, and elemental composition of formed products in the form of $\mathrm{Ti}_{3} \mathrm{AlC}_{2}$ and $\mathrm{TiC}$ are studied.
\end{abstract}

Keywords: high-power pulsed electron beam, self-propagating high-temperature synthesis.

DOI: $10.1134 /$ S0010508218020041

\section{INTRODUCTION}

Ignition and subsequent combustion of multicomponent condensed systems is a complex multistep process, which has various physical and chemical stages caused by the existence of zones in various aggregate states, as well as the possibility of chain, sequential, or parallel reactions, unequally significant in different zones [1-3]. It was shown in [3-6] that, in one and the same multicomponent condensed system with the course of competing processes under the same conditions, there could be several stationary combustion wave modes, whose implementation is uniquely determined by the ignition conditions.

${ }^{a}$ Prokhorov General Physics Institute, Russian Academy of Sciences (GPI RAS), Moscow, 119991 Russia; carbin@yandex.ru.

${ }^{b}$ Tomsk Polytechnic University, Tomsk, 634050 Russia; ep.gvp@yandex.ru

${ }^{c}$ Department of Structural Macrokinetics, Tomsk Scientific Center, Russian Academy of Sciences, Tomsk, 634021 Russia.

${ }^{d}$ Belgorod State University, Belgorod, 308015 Russia.

${ }^{e}$ Institute of Problems of Chemical Physics, Russian

Academy of Sciences, Chernogolovka, 142432 Russia.

${ }^{f}$ Institute of High Current Electronics (IHCE),

Siberian Branch, Russian Academy of Sciences,

Tomsk, 634055 Russia.
The processes of self-propagating high-temperature synthesis (SHS) in $\mathrm{M}_{n+1}-\mathrm{A}-\mathrm{X}_{n}$ ternary systems, initiated by various heat sources (electric spiral, laser, etc.) are described in sufficient detail in $[7,8]$.

Considerable scientific and practical interest is given to the ignition of ternary systems by a high-power electron beam and the development of the experimental technique with the stabilization of optimal parameters of the beam for SHS. Accelerators with high-power beams of charged particles are accessible today [9-14] and used to conduct multiple experiments associated with modifying the properties of various materials.

In this paper, the "SOLO" electron-beam device [12] is used to carry out experiments with stimulation of ternary exothermic mixtures by a high-power pulse electron beam in vacuum.

\section{METHODS AND MATERIALS}

The experiments are carried out using commercial powders, whose properties are described in Table 1 ( $D$ is the fraction size, $\mu$ is the molar mass, $T_{m}$ is the melting point, $Q$ is the latent heat of melting, and $K$ is the number of moles of components calculated for obtaining the compound $\mathrm{Ti}_{3} \mathrm{AlC}_{2}$, all of which are taken from [15]). 
Table 1. Characteristics of the powders used

\begin{tabular}{c|c|c|c|c|c|c}
\hline Element & Powder type & $D, \mu \mathrm{m}$ & $\mu, \mathrm{g} / \mathrm{mol}$ & $T_{m},{ }^{\circ} \mathrm{C}$ & $Q, \mathrm{~kJ} / \mathrm{mol}$ & $K$ \\
\hline $\mathrm{Ti}$ & PTS & $\approx 150$ & 48 & 1668 & 15.5 & 3 \\
\hline $\mathrm{Al}$ & ASD-4 & $10-50$ & 27 & 660 & 10.8 & 1.1 \\
\hline $\mathrm{C}$ & PM15 & $0.3-0.5$ & 12 & 4500 & - & 1.8 \\
\hline
\end{tabular}

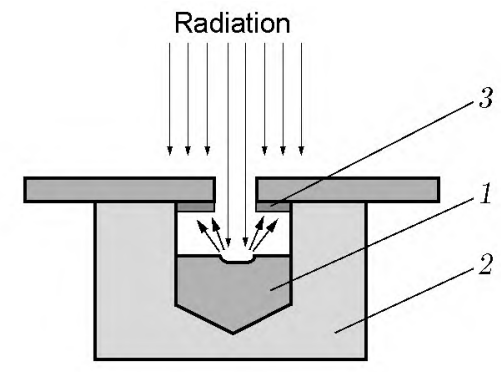

Fig. 1. Experimental diagram for the irradiation of Ti-Al-C system: (1) Ti-Al-C powder mixture pellet: (2) graphite crucible; (3) aperture.

Figure 1 shows the experimental diagram for the synthesis of the SHS products using an electron beam. $\mathrm{A} \mathrm{Ti}-\mathrm{Al}-\mathrm{C}$ powder mixture pellet $(15 \mathrm{~mm}$ in diameter, $10 \mathrm{~mm}$ in thickness, $5 \mathrm{~g}$ in mass, and with $40 \%$ of porosity) are placed in a graphite crucible $20 \mathrm{~mm}$ in height with a blind hole $15 \mathrm{~mm}$ in diameter and covered by a copper diaphragm with a $\approx 5$-mm hole for the passage of an electron beam into the crucible. The crucible is placed in a vacuum chamber with an electronic source with a plasma cathode.

An electron beam is used with the energy $E_{0}=15 \mathrm{keV}$, pulse duration $\tau=200 \mu$ s, repetition rate of $1 \mathrm{~Hz}$, and practically uniform distribution of the density flux along the cross section of the beam, whose dimensions on the pellet with an area of $0.2 \mathrm{~cm}^{2}$ are determined by the input diaphragm (see Fig. 1). The energy density measured by the calorimeter at the entrance to the crucible is $\approx 40 \mathrm{~J} / \mathrm{cm}^{2}$ with the heat flux in the spot on the pellet is equal to $\approx 2 \cdot 10^{5} \mathrm{~W} / \mathrm{cm}^{2}$.

The weight of the samples before and after treatment is measured on an LV-210-A analytical scale.

The structure and elemental composition of the treated samples are studied on a FEI Quanta 600 electron microscope. The phase composition of the materials obtained is determined on a DRON-2 diffractometer with $\mathrm{Co} K_{\alpha}$ radiation in the Bragg-Brentano focus mode.

\section{EXPERIMENTAL RESULTS AND DISCUSSION}

Gas- and hydrodynamic processes and chemical transformations occurring at the stage of ignition can be understood if there is a clear idea of the space-time pattern of the interaction of the high-power electron flux with the $\mathrm{Ti}-\mathrm{Al}-\mathrm{C}$ porous heterogeneous system characterized by varying dispersiveness of the components.

The energy is pumped by an electron beam through the electron subsystem of an irradiated body, and the energy exchange with the phonon subsystem occurs through a characteristic time equal to the relaxation

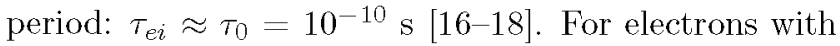
the energy $E_{0} \leqslant 300 \mathrm{keV}$, the main mechanism of electron absorption is the Coulomb scattering. At the same time, two-thirds of the beam energy goes to the excitation of bound electrons and the rest to ionization.

If the inflow rate of the energy (in $\mathrm{W} / \mathrm{cm}^{2}$ ) is comparable or larger with respect to the heat sink rate, the metal melts and subsequently evaporates due to the thermal conductivity in a solid in the energy release zone. The depth of the energy release zone is determined by the mean free path $R$ of the electron of the given energy in a particular substance $(\mathrm{Ti}, \mathrm{Al}, \mathrm{C}$, and gas). The dimensions of the energy release zone can be estimated by using the Kanaya-Okayama equation [19]

$$
R=2.76 \cdot 10^{-2} \mu E_{0}^{1.67} / Z^{0.89} \rho[\mu \mathrm{m}],
$$

where $\rho\left[\mathrm{g} / \mathrm{cm}^{3}\right]$ is the density of the irradiated substance, and $Z$ is the atomic number. Table 2 illustrates the values of $Z$ along with the values of the thermal diffusivity of the irradiated materials $a_{i}$, of the binding energy of the elements $\varepsilon$ [16], and of the threshold flux densities $q_{c}$, for which the materials fracture (evaporation and removal in a liquid phase) $[18,20]$. The study of the dynamics of the interaction of high-current electron beam with transparent low-density aerogel by optical methods showed that the size of the bulk energy release zone corresponds to the free path of electrons of a given energy in a particular substance and remains unchanged with constant parameters of the electron beam [14]. 
Table 2. Thermophysical parameters of the mixture elements

\begin{tabular}{c|c|c|c|c|c|c|c}
\hline Element & $Z$ & $E$, eV/atom & $\rho, \mathrm{g} / \mathrm{cm}^{3}$ & $R, \mu \mathrm{m}$ & $q_{c}, \mathrm{~W} / \mathrm{cm}^{2}$ & $\Delta H, \mathrm{~kJ} / \mathrm{kg}$ & $a, \mathrm{~m}^{2} / \mathrm{s}$ \\
\hline $\mathrm{C}$ & 6 & 7.36 & 3.5 & 3.5 & - & 59 & $1.22 \cdot 10^{-3}$ \\
\hline $\mathrm{Al}$ & 13 & 3.34 & 2.7 & 2.8 & $2 \cdot 10^{5}$ & 10.8 & $8.42 \cdot 10^{-5}$ \\
\hline $\mathrm{Ti}$ & 22 & 4.86 & 4.5 & 1.4 & - & 9.8 & $8.30 \cdot 10^{-6}$ \\
\hline
\end{tabular}

The sizes of the $\mathrm{Al}$ and $\mathrm{Ti}$ fractions (see Table 1) are larger than the free paths $R$ of the electrons with the energy $E_{0}=15 \mathrm{keV}$ (see Table 2 ) by more than an order of magnitude, and the diameters of soot (carbon) particles are an order of magnitude smaller than the free path $R_{\mathrm{C}}$. The initial surface of the $\mathrm{Ti}-\mathrm{Al}-\mathrm{C}$ pellet, on which the beam falls, has the $\mathrm{Ti}, \mathrm{Al}$, and $\mathrm{C}$ elements distributed mosaically thereon, and the irradiated heterogeneous body is a mixture of particles with varying dispersity and orientation. Assuming that the pellet pores containing $\mathrm{CO}, \mathrm{H}_{2}, \mathrm{O}_{2}$ and $\mathrm{N}_{2}$ [21] are the same in size with the carbon fraction, we can calculate the effective thermal diffusivity of the mixture $\left(a \approx 0.5 a_{\mathrm{Ti}}+0.31 a_{\mathrm{C}}+0.19 a_{\mathrm{Al}}\right)$ with a given porosity of $40 \%[22]$ and determine the size of the thermal effect zone $\left(L_{h}\right)$ in condensed matter under the action of the electron beam with given parameters. The layer of the heated heterogeneous substance (thermal effect zones) increases for the radiation time $\tau$ in accordance with the equation

$$
L_{h}=\sqrt{a \tau}
$$

$[17,18,20]$. If the energy of the electron beam equals to tens of kiloelectronvolts and the density created by the electron beam is $q_{0} \geqslant 10^{4} \mathrm{~W} / \mathrm{cm}^{2}$, the processes of evaporation and fracture of the surface of the processed material become essentially important [18, 20, 23].

The total thermal balance of the thermal effect zone in this case can be estimated in the expression

$$
Q_{e}+Q_{r}=Q_{s}+Q_{f}+Q_{v},
$$

where $Q_{e}$ is the energy introduced by the electron beam, $Q_{r}$ is the thermal effect of exothermic reactions, $Q_{s}$ is the heat flux caused by the thermal conductivity mechanism, and $Q_{f}$ and $Q_{v}$ are the heats of melting and evaporation, respectively.

If, during the irradiation time, the layer of the material $L_{h}$ obtains the energy comparable to the specific heat of evaporation, the evaporation process becomes governing [23]:

$$
q_{\mathrm{cr}} \sim \Delta H \rho L_{h} .
$$

This condition for the electron beam with a critical heat flux (specific density) $q_{\mathrm{cr}} \approx q_{0} \approx 2 \cdot 10^{5} \mathrm{~W} / \mathrm{cm}^{2}$ is satisfied. In the absorbing layer of the $\mathrm{Ti}-\mathrm{Al}-\mathrm{C}$ pellet, there is the phase transition of a condensed substance into a

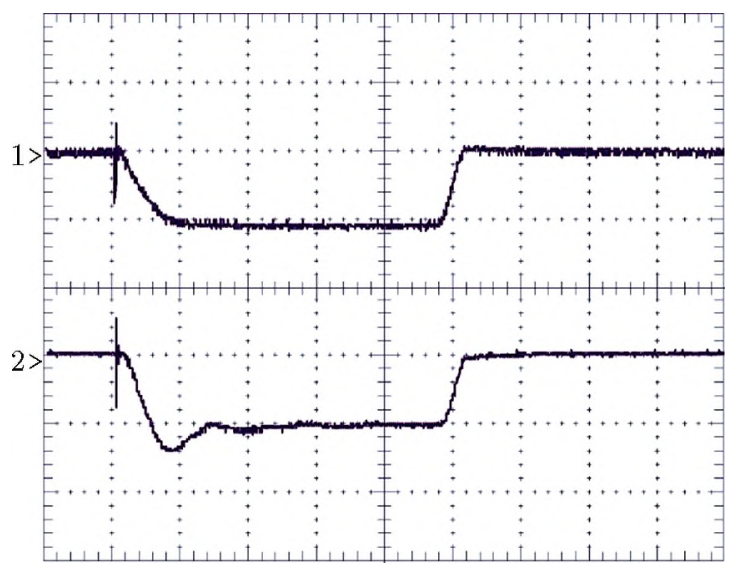

Fig. 2. Current oscillograms of the "SOLO" device [10]: (1) discharge current $I_{d} ;(2)$ electron beam current $I_{g}$; the abscissa axis is $50 \mu \mathrm{s} / \mathrm{div}$, and the ordinate axis is relative units per division.

gaseous one, and the heat conduction mechanism determines the temperature distribution in the condensed phase only behind the moving boundary of evaporation $[18,20]$. If $Q_{s} \gg Q_{r}$ in the layer, there is no stable combustion of the mixture and no combustion wave. The temperature profile of the combustion wave becomes observable only in the region where $Q_{s}<Q_{r}$.

In this experiment, the effect of a single electron pulse (Fig. 2) on the pellet of the ternary mixture ends with a bright flash (optical radiation is recorded with a high-speed camera) and a pressure jump in the accelerator chamber.

The melting process followed by evaporation is triggered by the leading edge of the pulse radiation. The trailing edge of the pulse $\approx 10 \mu$ s (see Fig. 2), in turn, triggers the high-rate solidification process.

The cylindrical pellet turns into a thick-walled shell after being treated with an electron beam, which is illustrated in Fig. 3. Explosion-like phase transitions in the exothermic porous system under the action of a high-power electron beam lead to the removal of the substance from the sample, reaching $60 \%$ of the initial mass, to the formation of regions with different surface topographies, and to an increase in the volume of the condensed object by approximately 2.5 times. 


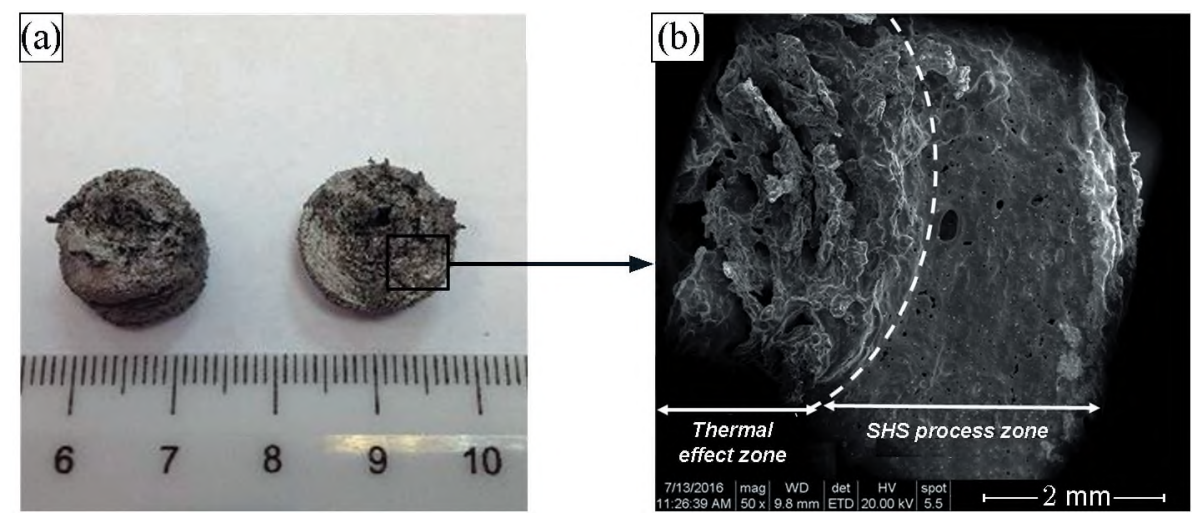

Fig. 3. General view (a) and structure (b) of the Ti-Al-C samples after treatment by a high-power electron beam.

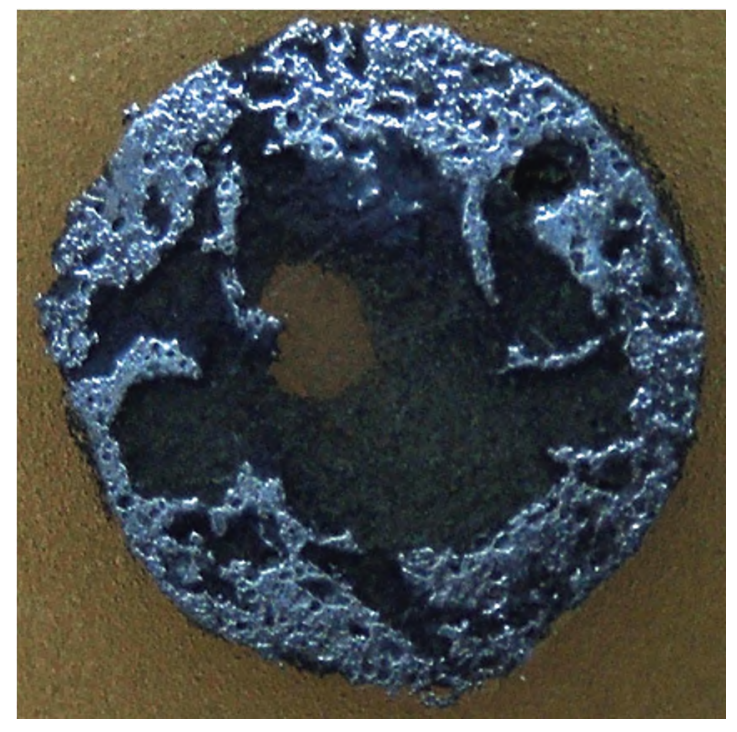

Fig. 4. Frontal view of the bottom part of the treated pellet.

To study the structure and elemental composition of the formed substance, the sample is cut approximately along the boundary between the top foamed part (gel) and bottom part [SHS product (dashed curve in Fig. 3b)].

The top part of the sample is significantly different from the bottom one (Fig. 4), which looks like a standard SHS product. There is a through hole with an incorrect shape, which is a canal formed by the shockwave effect of the electron beam. The effect of the beam lead to dagger penetration, almost complete removal from the top part of the aluminum sample, and partial removal of titanium and carbon. The removal of the substance matter from the sample volume is associated with high-rate evaporation and formation of $\mathrm{CN}, \mathrm{C}_{2} \mathrm{~N}_{2}$,

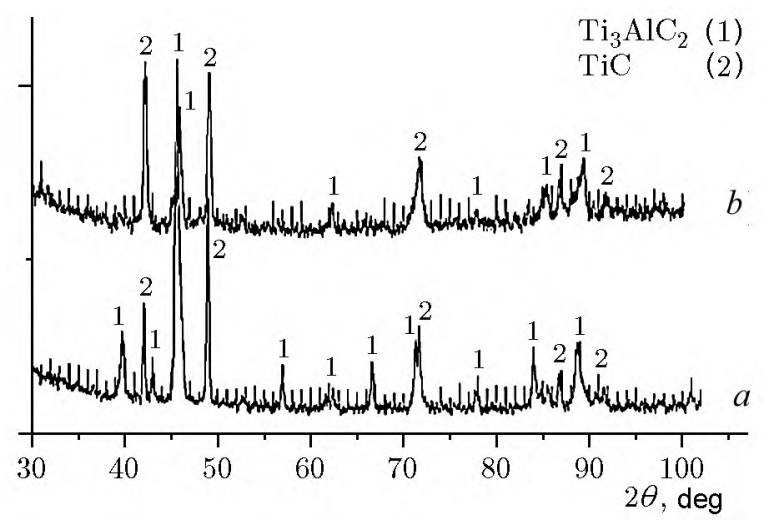

Fig. 5. Diffractograms of the SHS products: ignition of the mixture by electromagnetic radiation in an argon medium at a pressure of 5 atm (curve $a$ ) and ignition of the mixture by a high-power electron beam in vacuum (curve $b$ ).

$\mathrm{C}_{4} \mathrm{~N}_{2}, \mathrm{CO}$, and $\mathrm{CO}_{2}$ gases. The density of the formed product in the top part of the sample (see Fig. 3b) is noticeably smaller than in the bottom one (see Fig. 4).

The specific energy released during the time of the radiation pulse in the energy release zone $\left(\approx R_{c}\right)$, exceeds the binding energy of atoms in the elements by several orders of magnitude (see Table 2) [16, 24], so the energy input of the chemical reactions in this zone is insignificant. The ignition of the SHS process itself in the strongly nonequilibrium $\mathrm{Ti}-\mathrm{Al}-\mathrm{C}$ system occurs outside the thermal effect zone $\left(L_{h} \approx 0.5 \mathrm{~mm}\right.$ ) (see the bottom part in Fig. 3 and cylinder walls in Fig. 4).

The power in the spot on the pellet produced by the high-power electron beam and equal to $q_{0} \approx 2 \times$ $10^{5} \mathrm{~W} / \mathrm{cm}^{2}$ exceeds the standard one $[2,25] q=(1-2)$. $10^{2} \mathrm{~W} / \mathrm{cm}^{2}$ by three orders of magnitude and indicates that the ignition occurs in the region of the right branch 

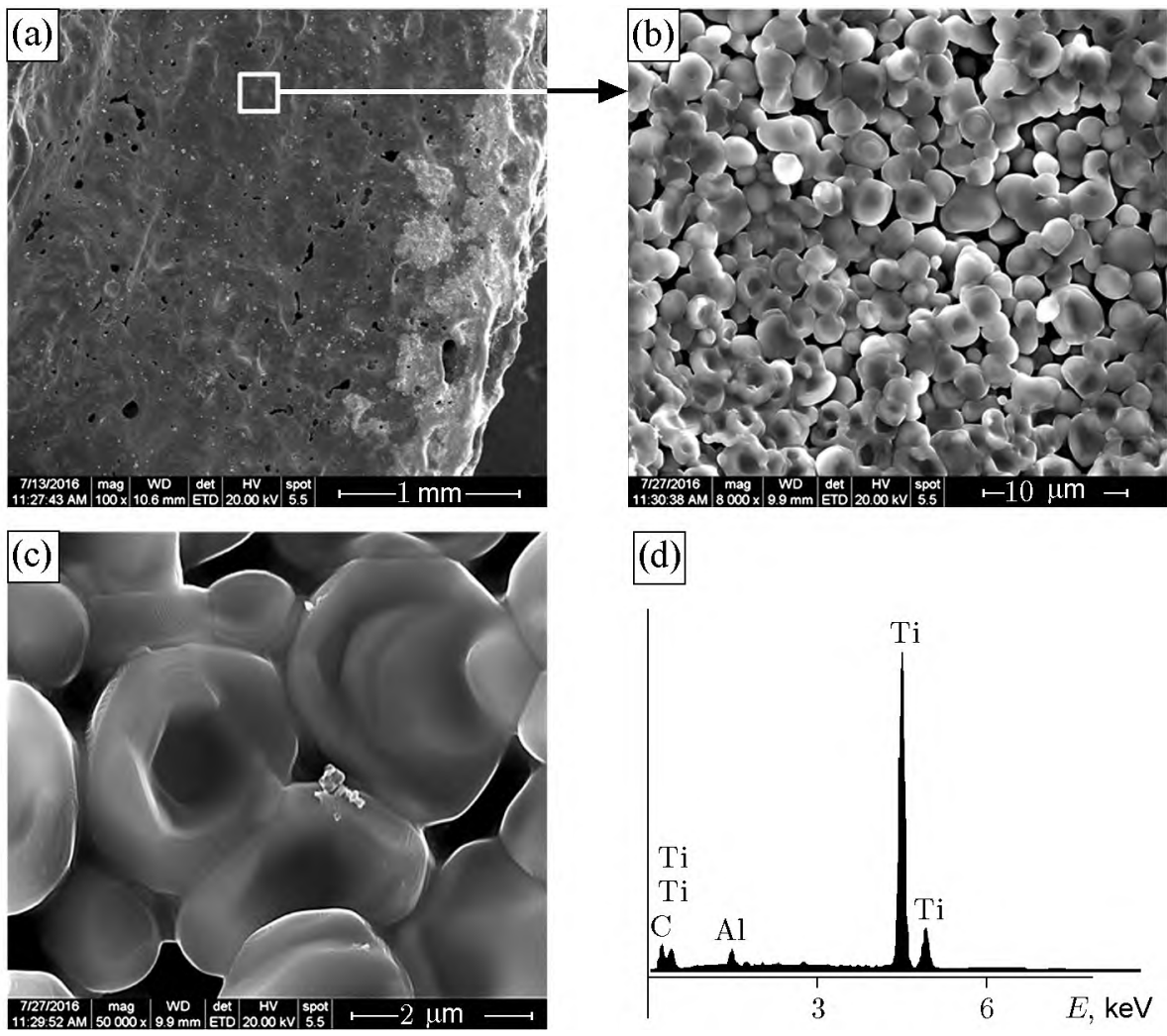

(d)

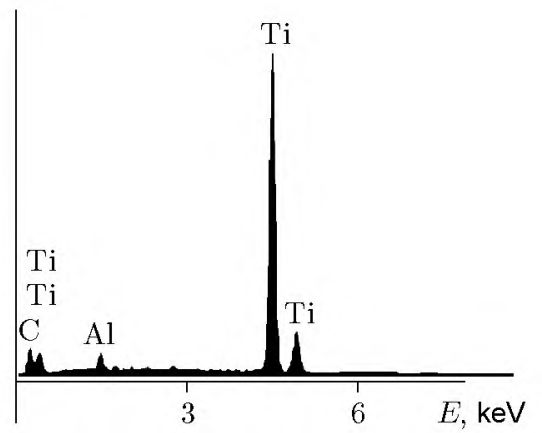

Fig. 6. Structure of the peripheral region (SHS process zones) of the Ti-Al-C sample after treatment with an electron beam: (b, c) morphological features of TiC crystals at different zooms; (d): $26.64 \%$ CK, $3.84 \%$ AlK, and $69.52 \%$ TiK.

of the curve illustrating the igniting pulse versus the density of the heat flux [24], when the temperature at the boundary of the phase transition reaches a value close to a boiling point of the material $T_{b}[18,20,25]$. The duration of the ignition delay determined by the video recording is $\approx 0.1 \mathrm{~s}$, the duration of the bright flash (SHS process) is equal to $\approx 4 \mathrm{~s}$, and the duration of the glow of the cooling SHS product is $10-12 \mathrm{~s}$.

With high-speed energy supply to the irradiated target, compression and rarefaction waves are formed therein. The appearance of a rarefaction wave can also lead to the release of substance from the irradiated target in a direction opposite to the direction of the electron beam $[13,14,26]$.

The diffractograms of the SHS products illustrated in Fig. 5 with the use of various ignition methods show different ratios of $\mathrm{Ti}_{3} \mathrm{AlC}_{2}$ and $\mathrm{TiC}$. According to the data of the quantitative $\mathrm{x}$-ray phase analysis, the sample $a$ contains $\approx 75 \%$ of $\mathrm{Ti}_{3} \mathrm{AlC}_{2}$ and $25 \%$ of $\mathrm{TiC}$ and the sample $b$ treated by the electron beam contains $18 \%$ of $\mathrm{Ti}_{3} \mathrm{AlC}_{2}$ and $82 \%$ of TiC. Such a difference in the phase ratio can be explained by the conditions for the SHS initiation of in these samples.
The composition of the substance in the top part of the sample treated with the electron beam (see Fig. 5) does not show the x-ray amorphous fraction of the product in the form of amorphous $\mathrm{Ti}$ and ultrafine phases of other compounds [27], which are present in the solidification of the melt with a temperature gradient of $10^{6} \mathrm{~K} / \mathrm{cm}$ and velocities of the interface boundaries of the order of $1 \mathrm{~m} / \mathrm{s}$ [28].

In the case of irradiation by a high-power electron beam, the $\mathrm{TiC}$ phase predominates due to the intense evaporation of the low-melting component of $\mathrm{Al}$. When the thermal energy of atoms of the irradiated material, obtained from the high-power electronic beam, decreases to a value comparable to the energy of interacting particles in the exothermic reaction, the SHS process begins in the target. Spatially, it is realized outside the thermal effect zone, and titanium carbide identified in this zone is not a product of SHS. In the temporary scale radiation, the $\mathrm{TiC}$ synthesis occurs after the end of the pulse (a few milliseconds). Nanolaminates are the products of the SHS process and formed at the periphery of the sample, where part of aluminum is still retained, not evaporated, and not removed in the liq- 
uid phase under the action of the high-power electron beam.

With high-rate solidification of the metallocarbon melt, the resulting structure of titanium carbide with $\mathrm{Ti}_{3} \mathrm{AlC}_{2}$ inclusions is a low-density gel-like product. The microstructures shown in Figs. 6a-6c characterize the morphological features of the cylindrical surface of the shell (see Fig. 4). The data of the energy dispersion analysis (Fig. 6d) show that, in this region, there is almost no aluminum, and the structural elements are mainly represented by titanium carbide in the form of hollow hemispheres (Figs. $6 \mathrm{~b}$ and $6 \mathrm{c}$ ).

The complete picture of the processes occurring in exothermic ternary porous mixtures under the action of high-power electron beams is far from clear and needs further research.

\section{CONCLUSIONS}

Under the action of the high-power electron beam, the $\mathrm{Ti}-\mathrm{Al}-\mathrm{C}$ sample radically changes its shape and content - the pellet turns into an inverted shell with a bulging bottom.

Under the action of the electron beams with a power density of more than $10^{3} \mathrm{~W} / \mathrm{cm}^{2}$, there is a change in the elemental composition due to removal of low-melting components from the mixture.

The use of high-power electron beams is effective for obtaining spatially heterogeneous structures, different by porosity and phase composition in depth.

\section{REFERENCES}

1. A. G. Strunina, V. I. Ermakov, S. V. Kostin, et al., "Choice of Optimal Ignition Conditions for Hard-toIgnite Gasless Systems," Fiz. Goreniya Vzryva 17 (4), 3-9 (1981) [Combust., Expl., Shock Waves 17 (4), 351356 (1981)].

2. V. P. Stovbun, T. I. Kedrova, and V. V. Barzykin, "Ignition of Systems with High-Melting Reaction Products," Fiz. Goreniya Vzryva 8 (3), 349-354 (1972) [Combust., Expl., Shock Waves 8 (3), 279-283 (1972)].

3. Concepts of Development of SHS as a Field of Scientific and Technological Progress, Ed. by A. G. Merzhanov (Territoriya, Chernogolovka, 2003) [in Russian].

4. T. P. Ivleva, A. G. Merzhanov, and K. G. Shkadinskii, "A New Type of Nonuniqueness of Stationary Modes of the Combustion Wave Propagation," Dokl. Akad. Nauk SSSR 256 (4), 897-884 (1981).
5. E. A. Levashov, Yu. V. Bogatov, and A. A. Milovidov, "Macrokinetics and Mechanism of the SVS-Process in Systems on a Titanium-Carbon Base," Fiz. Goreniya Vzryva 27 (1), 88 -93 (1991) [Combust., Expl., Shock Waves 27 (1), 83-88 (1991)].

6. V. N. Vilyunov, Theory of Ignition of Condensed Matter (Nauka, Novosibirsk, 1984) [in Russian].

7. M. W. Barsoum and T. El-Raghy, "The MAX Phases: Unique New Carbide and Nitride Materials," Amer. Sci. 89 334-343 (2001).

8. E. A. Levashov, Yu. S. Pogozhev, D. V. Shtanskii, et al., "Self-Propagating High-Temperature Synthesis of Ceramic Materials on the Basis of $\mathrm{M}_{n+1} \mathrm{AX}_{n}$ in the TiCr-Al-C System," Izv. Vyssh. Uchebn. Zaved., Poroshk. Metallurg. Funkts. Pokr. 313 (2008).

9. A. V. Khaneft, "Criteria for Igniting Condensed Substances by an Electron Pulse," Khim. Fiz. 17 (8), 132 (1998).

10. B. P. Aduev, G. M. Belokurov, S. S. Grechin, et al., "Detonation of PETN Single Crystals Initiated by an Electron Beam," Fiz. Goreniya Vzryva 46 (6), 111-118 (2010) [Combust., Expl., Shock Waves 46 (6), 712-718 (2010)].

11. V. I. Korepanov, V. M. Lisitsyn, V. I. Oleshko, et al., "PETN Detonation Initiated by a High-Power Electron Beam," Technical Physics Letters 29 (8) [Pis'ma Zh. Tekh. Fiz. 29 (16), 23-28 (2003)].

12. S. V. Grigoriev, V. N. Devjatkov, N. N. Koval, et al., "The Automated Installation for Surface Modification of Metal and Ceramic-Metal Materials and Products by an Intensive Pulse Sub-Millisecond Electron Beam," in Proc. 9th Int. Conf. Modification of Materials with Particle Beams and Plasma Flows (Tomsk, Russia, 2126 September 2008).

13. V. A. Artemov, M. A. Vlasov, O. A. Malafeev, et al., "Experimental Study of Nonstationary Evaporation of Metal Under the Action of an Electron Beam," Zh. Tekh. Fiz. 48 (2), 192-195 (1978).

14. B. A. Demidov, V. P. Efremov, M. P. Ivkin, et al., "Determination of the Energy Release Profile in Aerogel," Zh. Tekh. Fiz. 67 (11), 26 (1997).

15. Y. Zou, Z. M. Sun, H. Hashimoto, et al., "Low Temperature Synthesis of Single-Phase $\mathrm{Ti}_{3} \mathrm{AlC}_{2}$ through Reactive Sintering Ti/Al/C Powders," Mater. Sci. Eng. A 473, 90-95 (2008).

16. C. Kittel, Introduction to Solid State Physics (Wiley, 1956).

17. A.V. Lykov and B. M. Smolskii, Heat and Mass Transfer (Energiya, Moscow, 1972; Jerusalem, Israel, 1967).

18. F. F. Vodovatov, A. A. Chel'nyi, V. P. Veiko, et al., Lasers in Technology (Energiya, Moscow, 1975) [in Russian]. 
19. K. Kanaya and S. Okayama, "Penetration and Energy Loss Theory of Electrons in Solid Targets," J. Phys., D. Appl. Phys. 5, 43-58 (1972).

20. N. N. Rykalin, A. A. Uglov, I. V. Zuev, Fundamentals of Electron-Beam Treatment of Materials (Mashinostroenie, Moscow, 1978) [in Russian].

21. V. F. Brekhovskikh, N. N. Rykalin, and A. A. Uglov, "On the Possible Effect of Gas Content in Metals on the Area Affect by a Laser Beam," Dokl. Akad. Nauk SSSR 190 (5), 1059 (1970).

22. A. Missenard, Conductivite Thermique des Solides, Liquides, Gaz et de Leurs Melanges (Editions Eyrolles, 1965).

23. Yu. V. Afanas'ev and O. N. Krokhin, Vaporization of Matter Exposed to Laser Emission (Zhurn. Eksp. Teoret. Fiz. 52 966-975 (1967) [J. Exptl. Theret. Phys. 52639 645 (1967).
24. A. A. Koval'skii, S. S. Khlevnoi, V. F. Mikheev, "The Ignition of Ballistite Powders," Fiz. Goreniya Vzryva 3 (4), 527-541 (1967) [Combust., Expl., Shock Waves 3 (4), 323-331 (1967)].

25. L. G. Strakovskii, P. I. Ulyakov, E. I. Frolov, "Role of Vaporization in Explosive Ignition," Fiz. Goreniya Vzryva 16 (6), 59-64 (1980) [Combust., Expl., Shock Waves 16 (6), 654-659 (1980)].

26. G. I. Kanel, S. V. Razorenov, A. V. Utkin, et al., Shock-Wave Phenomena in Condensed Media (Yanus-K, Moscow, 1996) [in Russian].

27. G. Nicolis and I. Prigogine, Self-Organization in Nonequilibrium Systems: From Dissipative Structures to Order Through Fluctuations (Wiley, 1977).

28. W. Kurz and D. J. Fisher, Fundamental of Solidification (Trans Tech Publications, 1986). 